\title{
ABOUT MODELLING OF THE GRAVITATIONAL FIELDS
}

\author{
Stanislav A. Podosenov \\ Russia 143363, Moscow \\ JaykovFoukzon \\ Israel, Tel Aviv \\ Elena R. Men'kova, \\ Russia 121354, Moscow
}

\begin{abstract}
Within the framework of the general theory of relativity $(G R)$ the modeling of the central symmetrical gravitational field is considered. The mapping of the geodesic motion of the Lemetr and Tolman basis on their motion in the Minkowski space on the world lines is determined. The expression for the field intensity and energy where these bases move is obtained. The advantage coordinate system is found, the coordinates and the time of the system coincide with the Galilean coordinates and the time in the Minkowski space.
\end{abstract}

\section{Keywords:}

Curvature tensor, Einstein, equations of structure, Eulerian coordinates, frames of eference, hypersurfaces, Kristoffel,Lagrangian coordinates, Lemetr,metric tensor, Miln,Minkowski, Riemann, Schwarzschild,space-time,Tolman,world lines.

\section{INTRODUCTION}

In physical comprehending of the solutions of the Einstein equations particularly in the case of the strong gravitational fields we inevitably have the difficulty of the solution interpretation. In the Riemannian space the radius-vector concept is absent and the time coordinate is not a time as in the Minkowsky space in the Galilean coordinates. Obviously for the physical interpretation of the GR results it is useful (if it is possible) to reformulate them on the special theory of relativity language in the plane space-time. The detail analysis of the GR difficulties is present in [1 -3]. In present work within the framework of the Einstein theory the attempt to map the geodesic motion of the probe particles in the Riemannian space on the motion on the world lines in the Minkowski space is made. Similar problem scope was considered in [4 - 6], but it was not solved definitively.

\section{General Modelling Statements}

Let us consider that in the Minkowski space $V_{4}$ with the signature $(+---)$ the continuum moves in some force field, the motion law of this continuum in the Lagrange variables has the form:

$$
x^{\mu}=x^{\mu}\left(y^{k}, \xi^{0}\right)
$$


where $x^{\mu}$ are the Euler coordinates, $y^{k}$ are the Lagrange coordinates constant along each fixed world line of the medium particle, $\xi^{0} / c$ is the some time parameter. The Greek indexes are changed from zero up to three, the Latin indexes are changed from unit up to three. We consider that the medium particles do not interact with each other and they interact only with the external field.

Similarly to electrodynamics [7] the actions for the probe particle in the force field we specify in the form

$$
S=-\int_{a}^{b} m c\left(d s+\alpha A_{\mu} d x^{\mu}\right), \quad \alpha \equiv \frac{e}{m c^{2}}
$$

where for each medium particle the $d s$ interval along the world lines is $d s=V_{\mu} d x^{\mu}, V^{\mu}$ is the four dimensional velocity.

From the action variation the motion equation follows [7]

$$
\frac{D V_{\mu}}{d s}=\alpha F_{\mu \nu} V^{v}
$$

where the field tensor $F_{\mu \nu}$ is determined as

$$
F_{\mu \nu}=\nabla_{\mu} A_{v}-\nabla_{v} A_{\mu}=\frac{\partial A_{v}}{\partial x^{\mu}}-\frac{\partial A_{\mu}}{\partial x^{v}}
$$

On the other hand one can to introduce the effective interval $d \tilde{s}=d s+\alpha A_{\mu} d x^{\mu}$ so (2) is represented in the form

$$
S=-m c \int d \tilde{s}
$$

variation of (2) results in the motion of the probe particle on the geodesic line in some Riemannian space [7]

$$
\frac{d U_{\mu}}{d \tilde{s}}+\tilde{\Gamma}_{\mu, \nu \grave{~}} U^{v} U^{\grave{\mathrm{o}}}=0
$$

Obviously, that the equations (3) and (6) have to be an equivalent.

It follows from the expression for the effective interval $d \widetilde{s}$ along the geodesic line that

$$
\begin{gathered}
d \tilde{s}=\left(V_{\mu}+\alpha A_{\mu}\right) d x^{\mu} \equiv U_{\mu} d x^{\mu}, \quad U_{\mu} \equiv V_{\mu}+\alpha A_{\mu}, \\
U^{\mu}=\frac{d x^{\mu}}{d \tilde{s}}=\frac{d x^{\mu}}{d s} \frac{d s}{d \tilde{s}}=P V^{\mu}, \quad P \equiv\left(1+\alpha A_{\mathrm{o}} V^{\grave{\mathrm{o}}}\right)^{-1} .(7)
\end{gathered}
$$


Besides the connection between covariant $U_{v}$ and the contra variant $U^{\mu}$ vectors of the 4-velocity in the Riemannian space has the form

$$
U_{v}=g_{v \mu} U^{\mu}=V_{v}+\alpha A_{v} .(8)
$$

Conditions (3), (4), (6), (7) and (8) will be simultaneous if the metric tensor of the Riemannian space $g_{\mu \nu}$ will have the form:

$$
g_{\mu v}=\gamma_{\mu v}+\alpha^{2} A_{\mu} A_{v}+\alpha A_{\mu} V_{v}+\alpha A_{v} V_{\mu},(9)
$$

where $\gamma_{\mu \nu}$ is the metric tensor in the Minkowski space.

Thus, one can consider the motion of the probe particle from two points of view:

1. The motion on the world line in the Minkowski space in the force field (3) with the metric $\gamma_{\mu v}$.

2. The motion in the Riemannian space on the geodesic line with the metrics $g_{\mu \nu}$ determined in accordance with (9).

The correlations between the 4-velocities in the different spaces are determined with the formulas (7) and (8). Herewith in the two spaces the general coordination has been selected. Unlike electrodynamics the field tensor structure $F_{\mu \nu}$ in (4) has not been concreted, that is for $F_{\mu \nu}$ the field equations are not specified.

Let the probe particles move in the gravitational field. Then the "charge" $e=m$, and the metric (9) has to satisfy to the Einstein equations with the dust-like pulse energy tensor.

$$
R_{\mu \nu}-\frac{1}{2} g_{\mu \nu} R=\frac{8 \pi k}{c^{4}} \grave{\mathrm{o}} U_{\mu} U_{v} .(10)
$$

If as a result of the solution of the equations (10) obtained $g_{\mu v}$ and $U_{v}$ will provide the equalities (8) and (9), then we can to find the field of 4-velocity $V_{\mu}$, the potentials $A_{\mu}$ and the field tensor $F_{\mu \nu}$ in the Minkowsky space, that is the mapping of the curvature field of the Riemannian space on the force field of the plane space-time will be constructed.

Let us ascertain the connection between the congruencies of the world lines in the Minkowskispace and the congruencies of the geodesic lines in the Riemannian space which in the general coordination are determined with the correlation (1). Because of the correlation (9) in the space-time two metric tensors $g_{\mu \nu}$ and $\gamma_{\mu \nu}$ have been introduced, and, consequently, two connectivities $\tilde{\Gamma}_{\mu \nu}^{\varepsilon}$ and $\Gamma_{\mu \nu}^{\varepsilon}$ exist, the first connectivity relates to the Riemannian space, and the second one relates to the Minkowski space. In the Minkowski space the curvature coordinates can 
be introduced. Thus, in the general coordination two different covariant derivatives $\tilde{\nabla}_{v}$ and $\nabla_{v}$ arise.

From the correlation (8) we have

$$
\begin{gathered}
\tilde{\nabla}_{\nu} U_{\mu}=-S_{\nu \mu}^{\grave{\mathrm{o}}} U_{\grave{\mathrm{o}}}+\nabla_{\nu} V_{\mu}+\alpha \nabla_{v} A_{\mu}, \\
S_{\nu \mu}^{\grave{\mathrm{o}}}=\tilde{\Gamma}_{v \mu}^{\grave{\mathrm{o}}}-\Gamma_{\nu \mu}^{\grave{\mathrm{o}}},(11)
\end{gathered}
$$

where $S_{v \mu}^{\varepsilon}$ is the tensor of the affine connectivity deformation. Integrating (11) we find

$$
2 \tilde{\nabla}_{[v} U_{\mu]}=2 \nabla_{[v} V_{\mu]}-\alpha F_{\mu v},(12)
$$

For geodesic congruences without rotations the equalities take place

$$
\tilde{\nabla}_{[\nu} U_{\mu]}=0 ; \quad 2 \nabla_{[v} V_{\mu]}=\alpha F_{\mu \nu} .(13)
$$

Convoluting (13) with $V^{v}$ we once again obtain the correlation (3). From the equalities (13) and (7) we have

$$
U_{\mu}=\frac{\partial \Phi}{\partial x^{\mu}}=V_{\mu}+\alpha A_{\mu},(14)
$$

that permits the representation of the (9) metric in the form

$$
g_{\mu v}=\gamma_{\mu v}+\frac{\partial \Phi}{\partial x^{\mu}} \frac{\partial \Phi}{\partial x^{v}}-V_{\mu} V_{v}
$$

For the contra variant components we have

$$
\begin{gathered}
g^{\mu v}=\gamma^{\mu v}+P^{2} V^{\mu} V^{v}\left(1+\gamma^{\alpha \beta} \frac{\partial \Phi}{\partial x^{\alpha}} \frac{\partial \Phi}{\partial x^{\beta}}\right) \\
-P\left(V^{\mu} \gamma^{\nu \sigma} \frac{\partial \Phi}{\partial x^{\sigma}}+V^{v} \gamma^{\mu \sigma} \frac{\partial \Phi}{\partial x^{\sigma}}\right),(16)
\end{gathered}
$$

where in accordance with (7)

$$
P=\left(1+\alpha \gamma_{\grave{\mathrm{o}} \sigma} A^{\grave{\mathrm{o}}} V^{\sigma}\right)^{-1}=\left(\frac{\partial \Phi}{\partial x^{\grave{\mathrm{o}}}} V^{\grave{\mathrm{o}}}\right)^{-1}=\left(\frac{d \Phi}{d s}\right)^{-1}
$$

It follows from the equalities (9), (14) and (15) 


$$
g_{\mu \nu}-U_{\mu} U_{v}=\gamma_{\mu \nu}-V_{\mu} V_{v}
$$

that is the projection operators determining the space geometry of the hypersurfaces orthogonal to the world lines in the Minkowski space and the hupersurfaces orthogonal to the geodesic lines in the Riemannian space are the invariants of the correspondence [8].

\section{Modeling OF THE SCHWARZSCHILD'SAND LEMETR METRICS}

Let us consider some particular cases of the mapping.

Let in the Minkowski space the dust continuum moves on the radius to the center. We consider the case of the stationary motion that means time independence of the velocity field in the Euler variables and the $A_{\mu}$ potentials. In the GR language this corresponds to the constant gravitational field.

In order to the metric tensor (15) does not obviously depend from the time and pass at the infinity to the Galilean form it is necessary that the velocity at the infinity becomes zero. Herewith the equalities have to fulfill

$$
\Phi=x^{0}+\Psi\left(x^{k}\right), \quad V_{a}=-V(r) n_{a}=-V(r) \frac{x_{a}}{r}
$$

Using formulas (15) and (19) we find the expressions for three-dimensional metric tensor $\tilde{\gamma}_{k l}=-g_{k l}+g_{0 k} g_{0 l} / g_{00} \quad ; \quad$ three-dimensional vector $\quad g_{1}=-g_{0 l} / g_{00}=-g_{0 l} / h$; three-dimensional antisymmetric tensor $f_{k l}=\partial g_{l} / \partial x^{k}-\partial g_{k} / \partial x^{l}$ [7]. As a result we have

$$
\begin{gathered}
g_{00}=h=1-V^{2}, g_{l}=n_{l} \frac{\frac{\partial \Phi}{\partial r}+V_{0} V}{h}, f_{k l}=0, V_{0}^{2}-V^{2}=1, \\
\tilde{\gamma}_{k l}=\delta_{k l}+D(r) n_{k} n_{l}, D \equiv \frac{2 V^{2}+2 V_{0} V \frac{\partial \Phi}{\partial r}+V^{2}\left(\frac{\partial \Phi}{\partial r}\right)^{2}}{1-V^{2}}, \tilde{\gamma}^{k l}=-g^{k l}=\delta^{k l}+T n^{k} n^{l}, n^{k}=n_{k}, V^{0}=V_{0} \\
\tilde{\gamma}^{k l} \tilde{\gamma}_{l n}=\delta_{n}^{k}, T=-\frac{2 V^{2}+2 V_{0} V \frac{\partial \Phi}{\partial r}+V^{2}\left(\frac{\partial \Phi}{\partial r}\right)^{2}}{\left(V^{0}+V \frac{\partial \Phi}{\partial r}\right)^{2}} .(20)
\end{gathered}
$$

Einstein equations for the case of the constant gravitational field in the vacuity (we consider that the dusty medium is strongly discharged and itself does not create the field) [7] will result to two independent expressions 
International Journal of Recent advances in Physics (IJRAP) Vol.4, No.1, February 2015

$$
\begin{gathered}
\frac{\partial}{\partial r}\left(\frac{r^{2} \frac{\partial F}{\partial r}}{\sqrt{1+D}}\right)=0, F \equiv \sqrt{h}=\sqrt{1-V^{2}} \\
D+\frac{r}{2} \frac{\partial D}{\partial r} \frac{1}{(1+D)}=\frac{r}{F} \frac{\partial F}{\partial r},(21)
\end{gathered}
$$

solution of which has the form

$$
D=\frac{r_{g} / r}{1-r_{g} / r}, F=\sqrt{g_{00}}=\sqrt{1-\frac{r_{g}}{r}}, r_{g} \equiv \frac{2 k M}{c^{2}}
$$

From correlations (20) and (22) we find zero and radial field components of the 4-velocity in the Minkovsky space in the Euler variables an also $\Phi$ function.

$$
\begin{gathered}
V_{0}=V^{0}=\left(1+\frac{r_{g}}{r}\right)^{1 / 2}, V^{1}=V=-\sqrt{\frac{r_{g}}{r}}, \\
V_{0}+V \frac{\partial \Phi}{\partial r}=V^{\text {̀ }} \frac{\partial \Phi}{\partial x^{\mathrm{o}}}=\frac{d \Phi}{d s}=1
\end{gathered}
$$

Thus, $\Phi / c=\tau=s / c$ coincides with the own time of the basis particles in the Minkowski and Riemann space.

It follows from (23), (7) and (14) that $\left(1+\alpha A_{\mu} V^{\mu}\right)=P^{-1}=1$, this results in the equality of the contra variant components of 4-velocities $U^{\mu}=V^{\mu}$ of the basis particles in the plane and curved space-time. Covariant components $U_{\mu}$ and $V_{\mu}$ are connected with the correlation (14) Integrating equation (23) for $\Phi$ taking into account (19) we find

$$
\Phi=c \tau=s=x^{0}+\frac{2}{3} r_{g}\left\{\frac{r}{r_{g}}+1\right\}^{3 / 2}-\frac{2}{3} \frac{r^{3 / 2}}{r_{g}^{1 / 2}} .
$$

Using (20), (23) and (24) we obtain the expression for the interval element of the "original" in the spherical Euler coordinates and time $T$ of the Minkowski space ("model") found earlier by the author from other considerations [8]. 
International Journal of Recent advances in Physics (IJRAP) Vol.4, No.1, February 2015

$$
\begin{gathered}
d \tilde{s}^{2}=c^{2} d T^{2}\left(1-\frac{r_{g}}{r}\right)-d r^{2}\left\{2\left[\frac{r}{r_{g}}\left(\frac{r}{r_{g}}+1\right)\right]^{1 / 2}-2 \frac{r}{r_{g}}+\frac{r_{g}}{r}\right\}+ \\
+2 c d T d r\left[\left(1+\frac{r}{r_{g}}\right)^{1 / 2}-\left(\frac{r}{r_{g}}\right)^{1 / 2}-\frac{r_{g}}{r}\left(1+\frac{r}{r_{g}}\right)^{1 / 2}\right]-r^{2}\left(\sin ^{2} \Theta d \varphi^{2}+d \Theta^{2}\right) .
\end{gathered}
$$

Known the field of the 4-velocity in the Euler variables we find the motion law of the continuum in the Lagrange variables (1) selecting as a time parameter $\xi^{0}$ the propertime $\tau=\Phi / c=s / c$. From (23) we have $d r / d s=V=-\left(r_{g} / r\right)^{1 / 2}$. Integrating we obtain $R-s=2 / 3\left(r^{3 / 2} / r_{g}^{1 / 2}\right)$, where $R$ is the constant of integration.

Taking into account (24) as a result we find

$$
\begin{gathered}
r=\left[\frac{3}{2}(R-c \tau)\right]^{2 / 3} r_{g}^{1 / 3}, \\
x^{0}=c T=R-\frac{2}{3} r_{g}\left\{\left[\frac{3}{2 r_{g}}(R-c \tau)\right]^{2 / 3}+1\right\},(26)
\end{gathered}
$$

that determines the sought motion law in the Lagrange variables, substitution of this law to the expression (25) results in the Lemetr interval element [7].

Formulas (23), (26) determine the kinematics of the dust-like medium moving with the acceleration on the radius to the center in the Minkowski space in the gravitational field of the central body. For the field of the three-dimensional velocity $v$, 4-acceleration $g$, three-dimensional acceleration $a$ and the three-dimensional force $N$ we have:

$$
\begin{gathered}
\frac{d r}{d T}=v=-c\left(1+\frac{r}{r_{g}}\right)^{-1 / 2}, \frac{1}{c^{2}} \frac{d^{2} r}{d \tau^{2}}=g=-\frac{r_{g}}{2 r^{2}}, \\
a=\frac{d^{2} r}{d T^{2}}=-\frac{c^{2} r_{g}}{2 r^{2}}\left(1+\frac{r_{g}}{r}\right)^{-2}, \\
N=\frac{d}{d T}\left(\frac{m v}{\left(1-\frac{v^{2}}{c^{2}}\right)^{1 / 2}}\right)=-\frac{m r_{g} c^{2}}{2 r^{2}\left(1+r_{g} / r\right)^{1 / 2}} .(27)
\end{gathered}
$$

Movement of the Lemetr basis in the Minkowski space is described with the functions continuous in the range $0<r<\infty$ not having the singularities at the gravitational radius. 
Three-dimensional velocity $v$ and three-dimensional acceleration $a$ are restricted at the origin of the coordinates, $v(0)=-c, a(0)=-c^{2} /\left(2 r_{g}\right)$. The value of the three-dimensional force $N$ (27) influencing on the probe mass from the side of the central body is smaller than in the Newton gravitation theory

$$
N=-\frac{k m M}{r^{2}\left(1+\frac{2 k M}{c^{2} r}\right)^{1 / 2}}
$$

It is evident the circumstance that the space components of the 4-velocity $c V^{1}$ (23) and 4-acceleration $g c^{2}$ (27) exactly coincide with the usual velocity and acceleration in the non-relativistic Newton mechanics, when the radial fall of the dust having zero velocity at the infinity on the force center is considered.

From the formulas (23) and (27) we find the time of the basis particles fall from the distance $r_{1}>r$ up to $r \geq 0$ in accordance with the clock of the falling particle $\tau$ and in accordance with the Minkowski space clock $T$ [8].

$$
\begin{gathered}
\delta \tau=\frac{2}{3}\left[\frac{r_{1}}{c}\left(\frac{r_{1}}{r_{g}}\right)^{1 / 2}-\frac{r}{c}\left(\frac{r}{r_{g}}\right)^{1 / 2}\right],(29) \\
\delta T=\frac{2}{3}\left[\left(1+\frac{r_{1}}{r_{g}}\right)^{3 / 2}-\left(1+\frac{r}{r_{g}}\right)^{3 / 2}\right] \frac{r_{g}}{c}
\end{gathered}
$$

Correlation (29) coincides with the result of the Newton theory and similar formula obtained from GR in [9].

It follows from the formulas (29), (30) that the time of the particle fall is finite for any $r$ from the range $0 \leq r \leq r_{1}$ both in accordance with the clock of the fallen particle and in accordance with the clock of the Minkowski space.

Usually in GR the time coordinate $t$ including in the Schwarzschild'ssolution is introduced as a time of the external observer. The connection between the $t$ coordinate and $T$ time of the Minkowski space is determined with the formula [8]

$$
\begin{gathered}
T=t-\frac{1}{c} \int\left[\left(1+\frac{r}{r_{g}}\right)^{1 / 2}\left(1-\frac{r_{g}}{r}\right)-\left(\frac{r}{r_{g}}\right)^{1 / 2}\right]\left(1-\frac{r_{g}}{r}\right)^{-1} d r= \\
t-\frac{r_{g}}{c}\left[\frac{2}{3}\left(1+\frac{r}{r_{g}}\right)^{3 / 2}-2\left(\frac{r}{r_{g}}\right)^{1 / 2}\left(1+\frac{1}{3} \frac{r}{r_{g}}\right)-\right. \\
\left.\ln \left|\frac{1-\left(r / r_{g}\right)^{1 / 2}}{1+\left(r / r_{g}\right)^{1 / 2}}\right|\right] .
\end{gathered}
$$


Substitution of the formula to the interval (25) forms the Schwarzschild's interval.

The velocity field of the Lemetr basis $d r / d t$ in the Schwarzschild'smetric is connected with the velocity field $d r / d T=v$ (4.21.27)in the Minkowski space with the correlation

$$
\frac{d r}{d t}=\frac{d r}{d T} \frac{d T}{d t}=\frac{d r}{d T}\left(\frac{\partial T}{\partial t}+\frac{\partial T}{\partial r} \frac{d r}{d t}\right)
$$

Whence using (31) we find

$$
\frac{d r}{d t}=\frac{\frac{d r}{d T} \frac{\partial T}{\partial t}}{1-\frac{d r}{d T} \frac{\partial T}{\partial r}}=-c\left(1-\frac{r_{g}}{r}\right)\left(\frac{r_{g}}{r}\right)^{1 / 2}
$$

that coincides with the "coordinate" parabolic velocity of the free fall in the Schwarzschild's field obtained from the equations for the geodesic [9]. If the "coordinate" velocity in the Schwarzschild'sfield goes to zero when approximation to the gravitational radius then the velocities of the particles in the Minkowski space in the force field (28) are always smaller than the light velocity in the vacuum and their tend to the light velocity when $r \rightarrow 0$, and at the gravitational radius $|v|=c / \sqrt{2}$.

It follows from (33) that if the external observer uses the time Schwarzschild'scoordinate as a time of the removed observer then the approximation to the gravitational radius demands the infinite value $t[7,9]$. The later becomes clear from the form of the formula (31) when at $r \rightarrow r_{g}, t \rightarrow 0$ at any finite $T$.

From our view point $T$ should be taken as the time of the removed observer, $T$ in accordance with the image construction is the time in the Minkowski space and interval (25) is written in the "primary" coordinate system where the radial $r$, angle $\Theta$ and time $T$ coordinates have evident metric sense and they determine the interval in the Minkowski space in the form

$$
d s^{2}=c^{2} d T^{2}-d r^{2}-r^{2}\left(\sin ^{2} \Theta d \varphi^{2}+d \Theta^{2}\right) .
$$

At $r_{g} / r<<1$ the interval element (25) passes to the interval of the plane space-time (34). Naturally besides interval (25) one can consider any other coordinate systems but from our view point the coordinates entering to (25) coincide with the STR Galilean coordinates and so they are stood out with their clarity from all other coordinate systems.

As is well known when moving the particle in the constant field its energy $W_{0}$ is kept, $W_{0}$ is the time component of the covariant 4-vector of the pulse [7].

From (14), (24) we have for the basis particles

$$
W_{0}=m_{0} c^{2} U_{0}=m_{0} c^{2}=m_{0} c^{2}\left(V_{0}+\alpha A_{0}\right)
$$

whence using (23), (24), (35), (19) we find 
International Journal of Recent advances in Physics (IJRAP) Vol.4, No.1, February 2015

$$
\begin{gathered}
\alpha A_{0}=1-\left(1+\frac{r_{g}}{r}\right)^{1 / 2}, \\
\alpha A_{k}=\frac{\partial \Phi}{\partial x^{k}}-V_{k}=\left[\left(1+\frac{r}{r_{g}}\right)^{1 / 2}-\left(\frac{r}{r_{g}}\right)^{1 / 2}-\left(\frac{r_{g}}{r}\right)^{1 / 2}\right] n_{k} .
\end{gathered}
$$

It follows from (36) that $\alpha A_{\mu} V^{\mu}=0$ that is in agreement with (23).

Thus, the solution of the Einstein equations determined the metric $g_{\mu v}(25)$ in the coordinates of the Minkowski space, field velocity $V_{\mu}$ and $A_{\mu}$ potentials. From (36) we find the tensor of the constant gravitational field $F_{\mu \nu}$ in the Minkowski space

$$
\begin{gathered}
F_{\mu \nu}=\left(\frac{\partial A_{v}}{\partial x^{\mu}}-\frac{\partial A_{\mu}}{\partial x^{v}}\right), \quad F_{k l}=0, \\
F_{0 k}=-\frac{\partial A_{0}}{\partial x^{k}}=-\frac{r_{g} n_{k}}{2 \alpha r^{2} \sqrt{1+\left(r_{g} / r\right)}} .
\end{gathered}
$$

Similarly to the electrodynamics one can see that the tensor $F_{\mu \nu}$ for the case of the spherical symmetry does not contain the analogue of the $\vec{H}$ "magnetic" field. The intensity of the gravitational field $E_{k}$ taking into account (28) has the form

$$
E_{k}=F_{0 k}=\frac{N}{m_{0}} n_{k}=-\frac{k M n_{k}}{r^{2}\left(1+\frac{2 k M}{c^{2} r}\right)^{1 / 2}}
$$

Let us introduce the "induction" vector $D_{k}=\varepsilon E_{k}$

$$
\varepsilon \equiv-\left(1+\frac{2 k M}{c^{2} r}\right)^{1 / 2} \frac{1}{k}, \quad D_{k}=\frac{M}{r^{2}} n_{k}
$$

Thus, for the case of the spherically-symmetrical gravistatic field outside of the creating mass the expressions are valid

$$
\vec{\nabla} \times \vec{E}=0, \quad \vec{\nabla} \cdot \vec{D}=0, \quad \vec{H}=0
$$

Whence the energy density of the gravistatic field $\rho$ in analogy with the electrostatics is calculated in accordance with the formula 
International Journal of Recent advances in Physics (IJRAP) Vol.4, No.1, February 2015

$$
\rho=\frac{E D}{8 \pi}=-\frac{k M^{2}}{8 \pi r^{4}\left(1+\frac{2 k M}{c^{2} r}\right)^{1 / 2}} .
$$

Energy density has no a singularity at the gravitational radius unlike similar expression obtained in [10]. Field energy $W$ outside of the sphere with the radius $r_{0}$ is determined with the correlation

$$
W=\int_{r_{0}}^{\infty} \rho 4 \pi r^{2} d r=-\frac{M c^{2}}{2}\left[\left(1+\frac{r_{g}}{r_{0}}\right)^{1 / 2}-1\right],
$$

which passes to the Newton expression $W=-\left(k M^{2}\right) /\left(2 r_{0}\right)$ at $r_{g} / r<<1$.

From the problems considered one can to make the following conclusions:

It is possible to compare the central spherically-symmetrical gravitational field in vacuum determined from the Einstein equations and some equivalent force field in the Minkowski space. If the motion of the Lemetr basis in the Einstein space takes place on the geodesic lines then the motion of the same basis in the Minkowski space occurs on the world lines. The expression for the field strength in which this basis moves has been found and the expression for the field energy has been obtained. Within the GR framework the primary coordinate system (25) has been found, the coordinates and the time of this system coincide with the Galilean coordinates and the time in the Minkowski space. The radial Schwarzschild'scoordinate $r$ is equivalent to the value of the radius-vector in the Minkovsky space and the time Schwarzschild'scoordinate t does not coincide with the time of the Minkowski space T. The coincidence takes place only for the $r_{g} / r<<1$ distances. Hereof the known paradox in GR is explained, in accordance with this paradox the "coordinate" particle velocity of the Lemetr basis tends to zero when approximation to the gravitational radius while the force effecting on the particles at $r \rightarrow r_{g}$ (from the GR standpoint) tends to the infinity.

Calculation of known GR effects in accordance with the metrics (25) connected with the path form results in the same result as in the Schwarzschild'sfield. The difference reveals in the expressions depending on the time and on the time derivatives.

For the light beams spreading on the radius from (25) at $d \widetilde{s}^{2}=0$ we have:

$$
\begin{gathered}
\left(\frac{d r}{d T}\right)_{1}=c_{1}(r)=c\left[1-\left(\frac{r}{r_{g}}\right)^{1 / 2}\right] \\
{\left[\left(1+\frac{r}{r_{g}}\right)^{1 / 2}\left(\left(\frac{r}{r_{g}}\right)^{1 / 2}-1\right)-\frac{r}{r_{g}}\right]^{-1}}
\end{gathered}
$$


International Journal of Recent advances in Physics (IJRAP) Vol.4, No.1, February 2015

$$
\begin{gathered}
\left(\frac{d r}{d T}\right)_{2}=c_{2}(r)=c\left[1+\left(\frac{r}{r_{g}}\right)^{1 / 2}\right] \\
{\left[\frac{r}{r_{g}}-\left(1+\frac{r}{r_{g}}\right)^{1 / 2}\left(\left(\frac{r}{r_{g}}\right)^{1 / 2}+1\right)\right]^{-1},(44)}
\end{gathered}
$$

where (43) corresponds to the velocity of the spreading beams, and (44) corresponds to the velocity of the converging ones

At $r<r_{g}$ the expressions (43), (44) are negative that is the beams spread only in one direction inside [7].

$$
c_{1}\left(r_{g}\right)=0 \text {. }
$$

So the time of the light signal spreading from $r=r_{g}$ up to $r_{0}>r_{g}$ tends to infinity.

$\left|c_{1}\right|_{r>r_{g}}>0 ;\left|c_{1}\right| \leq c$ sign of equality takes place at $r \rightarrow 0 ; r \rightarrow \infty$.

$c_{2}<0 ;\left|c_{2}\right| \geq c$ sign of equality is valid at $r \rightarrow 0 ; r \rightarrow \infty$.

$\left|c_{2}\right|$ has a maximum at the $r=3 r_{g}$ point.

$$
\left|c_{2}\left(3 r_{g}\right)\right|=\frac{c(7+3 \sqrt{3})}{11}
$$

For converging beams the time of the signal spreading between any $r_{1}$ and $r_{2}$ from the range $0 \leq r<\infty$ is finite.

If $r_{g} / r<<1$, then

$$
\begin{gathered}
c_{1} \approx\left(1-0.5\left(r_{g} / r\right)^{1 / 2}-r_{g} / r\right) c, \\
c_{2} \approx-\left(1+0.5\left(r_{g} / r\right)^{1 / 2}-r_{g} / r\right) c .
\end{gathered}
$$

Though in each spreading direction $d r / d T$ differs from $c$ on the value containing the correction of the first order, however obtained results do not contradict to the "fourth Shapiro effect" since the lag time of the radio signal $\Delta T$ at the section "there" + "back" coincides with the Schwarzschild $\Delta t$ in the Shapiro experiment.

On the basis of the analysis carried out the following effect can be predicted: 
The light velocity emitted from the earth perpendicular to the surface should be smaller than the velocity of light falling from the infinity normal its surface on $11.2 \mathrm{~km} / \mathrm{s}$, that corresponds to the second space velocity.

\section{Modeling of The Tolman Metrics}

Let us consider how the known Tolman solution [7] is mapped into the Minkovsky space. Using the law of the continuum motion (1), where $\xi^{0}$ is the some time parameter, the sense of the parameter will be determined later, we go in the Lagrange accompanying reference frame.

For the observers moving together with the medium the square of the space distance is:

$$
-d l^{2}=\left(\gamma_{\mu v}-V_{\mu} V_{v}\right) \frac{\partial x^{\mu}}{\partial y^{k}} \frac{\partial x^{v}}{\partial y^{l}} d y^{k} d y^{l} \equiv-\tilde{\gamma}_{k} l d y^{k} d y^{l}
$$

where $\left(\gamma_{\mu v}-V_{\mu} V_{v}\right)=\tilde{\gamma}_{\mu v}$ is the projection operator.

$$
V^{\mu}=\Theta \frac{\partial x^{\mu}}{\partial \xi^{0}}
$$

$V^{\mu}$ is the four - velocity, scalar $\Theta$ is determined from the normality condition $\gamma_{\mu \nu} V^{\mu} V^{v}=1$. Three-dimensional curvature tensor calculated in accordance with the (45) metrics depending on the vortex tensor and the tensor of the medium deformation velocities [11] in general case is differed from zero.

Let in the Riemannian space the dust-like matter moves "without rotations". In this case as is well known [7] the accompanying frame of reference will be the synchronous for which the square of the interval is

$$
d s^{2}=d \xi^{02}-\breve{\gamma}_{k l} d y^{k} d y^{l}
$$

In two different spaces of the "model" $V_{4}$ and original $\breve{V}_{4}$ we selected the general coordinates of the $x^{\mu}$ Euler and $y^{k}, \xi^{0}$ Lagrange.

Our approach to the modeling depends on the answer on the question. Does such $\breve{\gamma}_{k l}$ from (47) satisfying to the Einstein equations and determined from the equality (48) exist?

$$
\breve{\gamma}_{k l}=\tilde{\gamma}_{k l}=-\left(\gamma_{\mu v}-V_{\mu} V_{v}\right) \frac{\partial x^{\mu}}{\partial y^{k}} \frac{\partial x^{v}}{\partial y^{l}}
$$

In other words we demand the equality of the space distance in the "model" and in the "original" [8] that follows form (18).

Considering the radial motion of the dust in the spherical "model" coordinates we have for the interval (45) 
International Journal of Recent advances in Physics (IJRAP) Vol.4, No.1, February 2015

$$
d l^{2}=\left(V^{1} \frac{\partial x^{0}}{\partial R}-V^{0} \frac{\partial r}{\partial R}\right)^{2} d R^{2}+r^{2}\left(R, \xi^{0}\right)\left(\sin ^{2} \Theta d \varphi^{2}+d \Theta^{2}\right)
$$

where $r$ is the radial Euler coordinate, $R$ is the radial Lagrange coordinate. The angle $\Theta$ and $\varphi$ Euler and Lagrange variables coincide.

In the "original" the known Tolman solution [7] will be the solution of the equations of the central-symmetrical field in the accompanying frame of reference for the dust-like matter.

From the conditions (48) we find the equation for the modeling of the Tolman metric

$$
\frac{\partial r}{\partial \xi^{0}} \frac{\partial x^{0}}{\partial R}+\frac{\partial r}{\partial R}\left\{\left[\frac{\left(\frac{\partial x^{0}}{\partial \xi^{0}}\right)^{2}-\left(\frac{\partial r}{\partial \xi^{0}}\right)^{2}}{1+f(R)}\right]^{1 / 2}-\frac{\partial x^{0}}{\partial \xi^{0}}\right\}=0
$$

In the equation (50) the function $x^{0}\left(R, \xi^{0}\right)$ is considered as unknown. $r\left(R, \xi^{0}\right)$ is determined with the Tolman solution, $f(R)$ is the arbitrary function in this solution.

Let us consider some particular solutions of the equation (50).

a) If we select the proper time $\tau=s / c$ in the law of motion (1) as $\xi^{0} / c$ parameter then

$$
\left(\frac{\partial x^{0}}{\partial s}\right)^{2}-\left(\frac{\partial r}{\partial s}\right)^{2}=1,(51)
$$

In order to the equations (50) and (51) will be simultaneous it is necessary the satisfying the integrability conditions

$$
\frac{\partial^{2} x^{0}}{\partial R \partial s}=\frac{\partial^{2} x^{0}}{\partial s \partial R}
$$

that when using the Tolman solution

$$
\left(\frac{\partial r}{\partial s}\right)^{2}=f(R)+\frac{F(R)}{r}
$$

results in the relation

$$
\frac{d f}{d R}+\frac{1}{r} \frac{d F}{d R}=0
$$


Solution of the equation (53) is $f=c_{1}=$ const,$F=c_{2}=$ const . In particular the Lemetr metric [7] for which $f=o, F=r_{0}$ fulfills to this conditions.

Integration of the equation (50) results in the law of motion of the Lemetr basis particles in the "model" $V_{4}$ [8] obtained above (26).

b) Suppose in the equation (50) $\partial x^{0} / \partial R=0$ we have

$$
x^{0}=\Psi\left(\xi^{0}\right),\left(\frac{\partial r}{\partial \xi^{0}}\right)^{2}=-f\left(\frac{\partial \Psi}{\partial \xi^{0}}\right)^{2}, r=|f|^{1 / 2} \Psi .
$$

It follows from (54) that $f<0$. In particular if $f=-\sin ^{2} R, \Psi=a\left(\xi^{0}\right)$. From the formulas (46), (47 - 49) we find

$$
\begin{aligned}
& \Theta=\frac{1}{\sqrt{1+f}}\left(\frac{\partial x^{0}}{\partial \xi^{0}}\right)^{-1},\left(V^{0}\right)^{2}=\cos ^{-2} R,\left(\frac{\partial r}{\partial R}\right)^{2}=a^{2} \cos ^{2} R \\
& d \tilde{s}^{2}=\left(d \xi^{0}\right)^{2}-a^{2}\left(\xi^{0}\right)\left\{d R^{2}+\sin ^{2} R\left(d \theta^{2}+\sin ^{2} \theta d \varphi^{2}\right)\right\} .(55)
\end{aligned}
$$

Interval element (55) corresponds to the metric of the closed isotropic model [7].

It is important to point out that $a\left(\xi^{o}\right)=c T$, where $T$ is the time in the Minkowski space.

So in the "model" space the solution of the Einstein equation in the "original" is restricted with the time

$$
T_{\max }=\frac{2 a_{0}}{c}=\frac{4 k M}{3 \pi c^{2}} \frac{1}{c}
$$

Where $M$ is the mass of the closed model, $k$ is the gravitational constant

We obtain from (54) the velocity field of the basis particles in the "model" in the Euler and Lagrange variables

$$
\begin{gathered}
c \frac{\partial r}{\partial x^{0}}=v=\frac{r}{T}=c \sin R \leq c,(57) \\
\frac{d v}{d T}=0
\end{gathered}
$$

It is interesting to point out that in the considered case the "gravitational force" in the Galilean "model" space is equal to zero and the speed of "recession"at fixed $T$ time is proportional to $r$ (the Hebble law). The similar result has been obtained from other considerations by V. Fock [1].

The connection between the "model" time and the "original" time is expressed with the correlation 
International Journal of Recent advances in Physics (IJRAP) Vol.4, No.1, February 2015

$$
T=\frac{1}{c} a(t)=\frac{a_{0}}{c}(1-\cos \eta),(59)
$$

where following to [7] we introduced $c d t=a d \eta$. Denoting

$$
\frac{1}{T}=h_{1}, \quad \frac{1}{a} \frac{d a}{d t}=h
$$

we have

$$
h_{1}=h \tan \frac{\eta}{2}=h\left(\frac{\mu}{\mu_{c r}}-1\right)^{1 / 2}
$$

It follows form formula (60) that the "age" of the homogeneous closed universe model when the density $\mu \sim \mu_{c r}$ in accordance with the clock of the Minkowski space $1 / h_{1}$ and the "original" space $1 / h$ can noticeably differed from each other.

c) If in the law of motion (1) the parameter numbering the hypersurfaces orthogonal to the world lines has been selected as a time parameter [11] then

$$
V_{\mu} \frac{\partial x^{\mu}}{\partial y^{k}}=0,(61)
$$

whence it is follows that in the case of the spherical symmetry

$$
\frac{\partial x^{0}}{\partial R}=\frac{V^{1}}{V^{0}} \frac{\partial r}{\partial R}
$$

From formula (50) we find

$$
V^{0}=\sqrt{1+f}, \quad V^{1}=\sqrt{f}, \quad f>0 .(63)
$$

Integration (63) gives

$$
r(R, t)=\sqrt{\frac{f}{1+f}} x^{0}+B(R)=v T+B(R),(64)
$$

where $B(R)$ is the arbitrary function.

As it has been shown in [11] the vectors of the first curvature $g^{k}$ of the world lines of the medium particles in the Lagrange accompanying noninertial frame of reference (NRF) are connected with the normalizing factor $\Theta$ in (46) with the relation 
International Journal of Recent advances in Physics (IJRAP) Vol.4, No.1, February 2015

$$
g^{k}=\gamma^{k n} \frac{\partial \ln \Theta}{\partial y^{n}}
$$

In considered case $g^{k}=0$. So

$$
\Theta=\Theta\left(\xi^{0}\right)
$$

Taking into account this expression we find from (63) and (64) at $\mathrm{B}=0$

$$
\begin{gathered}
x^{0}\left(\xi^{0}, R\right)=a\left(\xi^{0}\right) \sqrt{1+f} \\
r\left(\xi^{0}, R\right)=a\left(\xi^{0}\right) \sqrt{f}, \quad \frac{1}{\Theta}=\frac{\partial a}{\partial \xi^{0}}
\end{gathered}
$$

In particular if $f=\sinh ^{2} R$ then we obtain for the interval element

$$
d \tilde{s}^{2}=\left(d \xi^{0}\right)^{2}-a^{2}\left(\xi^{0}\right)\left\{d R^{2}+\sinh ^{2} R\left(d \theta^{2}+\sin ^{2} \theta d \varphi^{2}\right)\right\},(68)
$$

that coincides with the metric of the open isotropic model [7].

Proposed modeling method permits within the GR framework clear up the metric sense of coordinates and the time expressing their viacoordinates and the time of the Minkowski space. Since

$$
V^{1}=\frac{d r}{d s}=\frac{d r}{c d \tau}
$$

Then

$$
a\left(\xi^{0}\right)=c \tau=\frac{c T}{\cosh R}
$$

Thus, $a\left(\xi^{0}\right) / c$ coincides with the proper time $\tau$ of the basis particles in the "model".

Let us introduce the Hebble "constant" in the "model" $h_{1}=1 / T$ and compare it with the value

$$
h=\frac{1}{a} \frac{d a}{d t}, \quad t=\frac{\xi^{0}}{c} .
$$

The comparison gives 


$$
h_{1}=h \sqrt{\frac{1-\frac{\mu}{\mu_{c r}}}{1+\frac{h^{2} r^{2}}{c^{2}}\left(1-\frac{\mu}{\mu_{c r}}\right)}} .
$$

When the $\mu$ density is closed to the critical density $\mu_{c r}$ the Universe has significantly greater age in accordance with the "model" clock then on the "original" clock.

As is well known the distance concept in the cosmology does not have unambiguous sense and the "correct" distances are absent [12]. Proposed method permits to consider the Euclidian distances $r=a\left(\xi^{0}\right) \sin R$ and $r=a\left(\xi^{0}\right) \sinh R$ for open and closed models accordingly as "correct".

Using known formulas [7]

$$
\begin{gathered}
a(\eta)=a_{0}(1-\cos \eta), \quad a_{0}=\frac{2 k M}{3 \pi c^{2}} \\
\mu a^{3}=\frac{M}{2 \pi^{2}}, \quad \xi^{0}=c t=a_{0}(\eta-\sin \eta)
\end{gathered}
$$

for closed model and formulas

$$
a(\eta)=a_{0}(\cosh \eta-1), \quad \mu a^{3}=\frac{3 c^{2} a_{0}}{4 \pi k}, \quad \xi^{0}=c t=a_{0}(\sinh \eta-\eta)
$$

for open model one can show that from the motion laws (54) $r(R, t)=a(t) \sin R$ and $r(R, t)=a(t) \sinh R$ the equality follows

$$
\frac{\partial^{2} r}{\partial t^{2}}=-\frac{4 \pi k \mu r}{3}
$$

Equality (71) coincides with the Newton law. We point out that in (71) the differentiation is realized on the proper time $t$ of the "original". The differentiation of the motion laws on the "model" time forms zero acceleration in the Minkowski space. Thus, in considered cosmology models the action of the gravitational field is revealed as the time deformations. The later statement is clear in the case when the interval (68) taking into account (69) and the parametric formulas for open model is presented in the form

$$
d \tilde{s}^{2}=\frac{c^{2} d \tau^{2}}{1+\frac{2 a_{0}}{c \tau}}-c^{2} \tau^{2}\left\{d R^{2}+\sinh ^{2} R\left(d \theta^{2}+\sin ^{2} \theta d \varphi^{2}\right)\right\}
$$

When the gravitation is absent $a_{0}=0$ and the interval element (72) coincides with the interval in the Miln model [9] realized with the particles flying from the one point on all directions with various velocities that is forming spherically symmetrical quasi-IRF [2] or generalized IRF [13]. 
International Journal of Recent advances in Physics (IJRAP) Vol.4, No.1, February 2015

Thus, the using of the modeling method in the cosmology showed that the connection between general theory of relativity (GR), special theory of relativity and the Newton's law of gravitation is more closed than usually proposed. If one calculates the age of the Universe in accordance with the Minkowski space clock then it follows from the formulas (60) and (70) that at the densities closed to the critical the Universe is significantly "older" of its "original" age.

\section{REFERENCES}

[1] V. A. Fock, "Theory of space time and gravitation," Ed. Moscow:Fizmatgiz, 1961.

[2] V. I. Rodichev, "Gravitational theory at the orthogonal frame," Ed. Moscow: Nauka, 1974.

[3] A.A.Logunov,"Lectures on theory of relativity and gravitation. Modern problem analysis,"Ed. Moscow: Nauka, 1987.

[4] N.Rosen, Physical Review,vol. 57, pp. 147-150, 1940.

[5] A. Z. Petrov, Preprint ITF - 71 - 130R, Ed. Kiev, 1971.

[6] N. S. Siniukov, "Geodesic mapping of Riemannian spaces," Ed. Moscow: Nauka, 1979.

[7] L. D. Landau, E. M. Lifshits, "Field Theory," Ed. Moscow: Nauka, 1973.

[8] S. A. Podosenov, "Modeling of centrosymmetricalgravitational field" in Heads of report4-thAll-UnionConferenceon modern theoretical and experimental problems of theory of relativity and gravitation, Minsk, BGU, 1976,pp. 258-260.

[9] Ia. B. Zel'dovich,I. D. Novikov, "Relativistic astrophysics," Ed. Moscow: Nauka, 1967.

[10] G. Denen, in "Einsteinian collection", Ed. Moscow: Nauka, 1969-1970, p. 140.

[11] S. A. Podosenov, "RectilinearBorn's rigidcontinuum motion with uniform acceleration in accompanying tetrad," in Problems of gravitation theory. Theoretical and Mathematical Physics, ser.Aissue 1, Ed. Moscow: VNIIOFI,1972,pp.95-104.

[12] G. K. Mak-Vitty, "General relativity theory and cosmology," Transl. from Engl., Moscow: Izd. Inostr. Lit., 1961.

[13] Kh. P. Keres, "Investigations on theoretical physics," Transactions IFA AN ESSR,20, Ed. Tartu: 1963. 